\title{
Estado del arte sobre narcoviolencia en Costa Rica
}

\section{State of the art on narcoviolence in Costa Rica}

\author{
Sebastián Saborío
}

Fecha de recepción: 23 de octubre de 2018

Fecha de aceptación: 23 de abril de 2019

\begin{abstract}
Resumen
En Costa Rica, el conocimiento sobre la narcoviolencia, es decir, la violencia vinculada al narcotráfico y al narcomenudeo, es escaso y muy fragmentado. Este artículo tiene el objetivo de mostrar cuál es, a nivel nacional, el estado del arte sobre este tema. Unificando el conocimiento existente sobre la narcoviolencia, se brinda un análisis y síntesis que pueden servir a aquellos que, desde el sector público y la sociedad civil, quieran entender cuáles son las características principales de dicho fenómeno, con el fin de buscar e implementar nuevas soluciones. En particular, se explica qué se entiende en el país por criminalidad organizada; qué sabemos sobre el impacto que el narcotráfico internacional tiene sobre el contexto nacional; cómo se organizan los grupos del narcomenudeo en las comunidades urbano-marginalizadas y cuáles son las causas sociales de este problema. En un segundo momento, son señalados los vacíos existentes en la literatura y, con base en estos se indican posibles rutas para futuras investigaciones académicas.
\end{abstract}

Palabras clave: Violencia, Narcotráfico, Narcomenudeo, Costa Rica, Estado de la cuestión.

\begin{abstract}
In Costa Rica the existing knowledge about narcoviolence, the violence related to drug trafficking and local drug-dealing, is sparse and very much fragmented. This article aims to show what is, at a national level, the state of the art on this topic. Unifying the existing knowledge on narcoviolence makes it possible to offer an analysis and synthesis that can be useful to those that, in the public sector and in civil society, want to understand what the main characteristics of this phenomenon are to then implement new solutions. In particular, we explain the country's understanding of organized crime, what we know about the impact that international drug trafficking has on the national context, how the local drug-dealing groups are organized in urban-marginalized communities, and what the social causes of this problem are. Secondly, the existing gaps in the literature are indicated, demonstrating possible routes for future academic research.
\end{abstract}

KeyWords: Violence, Drug Trafficking, Local Drug-dealing, Costa Rica, State of the art.

1. Instituto de Investigaciones Sociales / Escuela de Sociología, Universidad de Costa Rica, Costa Rica, sebastian.saborio@ucr.ac.cr

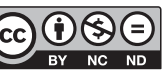




\section{Introducción}

Entre 1991 y 2005 en Costa Rica, se verificó un aumento de la tasa de homicidios, que pasó de 3.6 a 6.7 cada 100.000 habitantes (Mata y Solano Fernández 2006); tendencia que ha continuado hasta el 2017, año en el que se dio el número más alto de homicidios en la historia del país con 603 casos, lo que representa una tasa de 12,1cada 100.000 habitantes $^{1}$ (Sánchez Hernández 2018). Según las estadísticas oficiales de las denuncias ingresadas al Organismo de Investigación Judicial (OIJ), los homicidios que más han aumentado desde el 2010 han sido aquellos atribuibles a la criminalidad organizada, que pasaron de 0,3 cada 100.000 habitantes en ese año, a 5,5 en el 2016 (COMESCO 2017). Dichos homicidios parecen ser, sobre todo, el resultado directo del aumento de los conflictos entre organizaciones criminales relacionadas al narcotráfico y al narcomenudeo.

Este artículo presenta el estado del arte en Costa Rica sobre la narcoviolencia, entendiéndola como la «violencia relacionada al mercado de las drogas ilegales» (Parakilas 2012, 36), así como los elementos que la conforman y otros aspectos que pueden ayudar a comprenderla. A continuación, se presentarán los resultados obtenidos a partir de una recolección y análisis ${ }^{2}$ de la bibliografía existente, que se llevó a cabo consultando, en bases de datos académicas especializadas en ciencias sociales, las siguientes palabras clave: narcoviolencia, narcotráfico, narcomenudeo, drogas, homicidios, asesinatos, pandillas, bandas criminales, ajusticiamientos, ajustes de cuentas, violencia, violencia urbana, policía, prevención de la violencia, criminalidad y Costa Rica. En total, se identificaron las siguientes publicaciones académicas que analizan, o al menos mencionan, datos sobre el contexto nacional: 34 artículos de revistas científicas, 5 libros, 12 capítulos de libros, 3 working paper y 1 ponencia en una conferencia ${ }^{3}$. No se limitó la búsqueda y el análisis a los textos enfocados principalmente en los temas de interés de este artículo, sino que se intentó incluir también publicaciones que los mencionen de manera secundaria o que tratan elementos útiles a su comprensión. Inicialmente, la intención del autor era individuar los elementos y características propias del contexto nacional en relación al fenómeno analizado, así como demostrar cuáles son los vacíos existentes en la literatura académica para determinar las rutas que deben ser seguidas en las futuras investigaciones científicas. Sin embargo, para tener una mayor cantidad de datos, en un segundo momento se tomaron en consideración también $88^{4}$ publicaciones no académicas sobre estos temas, como por ejemplo informes de fundaciones y entes gobernativos.

\section{Crimen organizado}

Para López y Gómez Calderón, el narcomenudeo «consiste en la venta de dosis personales y de aprovisionamiento» $(2017,231)$, mientras que el narcotráfico está compuesto por: «producción, distribución, comercialización y capital circulante» (López y Gómez Calderón 2017, 31). Dicha definición permite diferenciar el narcomenudeo del narcotráfico y entender que este último no se reduce únicamente a la venta de drogas. El primer elemento a destacar es que muchas veces en las publicaciones analizadas se usa el término «narcotráfico» para referirse también al narcomenudeo.

La falta de diferenciación entre estas actividades en las publicaciones analizadas puede depender del hecho de que, desde el punto de vista legal, en Costa Rica se clasifica de la misma manera el narcotráfico y el narcomenudeo; y los pequeños grupos criminales son definidos y castigados como los grandes grupos de la criminalidad organizada internacional. En la Ley Contra la Delincuencia Organizada $N^{o} 8754$, se define una organización criminal como una: «Estructura creada y con vigencia ininterrumpida por cierto tiempo, compuesta por dos o más personas y actuando concertadamente con el propósito de cometer uno o varios delitos y a sabiendas de que estos se originan en un delito que, dentro de su rango de penas, puede ser sancionado con prisión de cuatro años o más» (ICD 2012, s.n.).

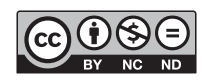

Rev. Reflexiones 98 (2) Julio-Diciembre, 2019: 23-38, E-ISSN: 1659-2859 
Mientras que la Ley Sobre Estupefacientes, Sustancias Psicotrópicas, Drogas de Uso no Autorizado, Actividades Conexas, Legitimación de Capitales y Financiamiento al Terrorismo homologa la gravedad de las siguientes actividades: «El comercio, el expendio, la industrialización, la fabricación, la refinación, la transformación, la extracción, el análisis, la preparación, el cultivo, la producción, la importación, la exportación, el transporte, la prescripción, el suministro, el almacenamiento, la distribución y la venta de drogas» (Asamblea Legislativa de la República de Costa Rica 1998, 2).

De esta manera, un narcomenudeante que vende pequeñas dosis de droga para el consumo personal en las calles de un barrio vulnerable, puede ser castigado con una pena que va de los 8 a los 15 años; es decir, de la misma manera que un narcotraficante internacional responsable del trasiego de grandes cantidades de narcóticos (ICD 2016). El 93,1\% de las incautaciones de droga realizadas en el territorio nacional por parte de la policía no superan los 5 gramos de droga (ICD 2017). Esto quiere decir que la represión no se focaliza principalmente en el narcotráfico internacional, sino en el consumo de drogas y en el narcomenudeo. El hecho de equiparar el narcomenudeo al narcotráfico, y catalogar ambas actividades como criminalidad organizada, reduce la posibilidad de comprender, por ejemplo, si el aumento de los homicidios que se está dando en Costa Rica, y que se mencionó hace poco, depende de conflictos entre grupos criminales que luchan por el monopolio de la venta de droga en localidades específicas o para controlar las rutas de entrada y salida de droga del país. La necesidad de diferenciar dichas actividades, no sólo desde el punto de vista legal sino también en las publicaciones académicas, está relacionada al hecho que, para comprender las causas y consecuencias sociales de la narcoviolencia e identificar las acciones necesarias para contrarrestarla, es necesario poder localizarla y saber las modalidades con las cuáles se genera y se reproduce.

\section{Narcotráfico}

Los resultados públicos de las investigaciones policiales nos indican que no siempre es fácil diferenciar las organizaciones que se dedican al narcomenudeo local de las que pertenecen al narcotráfico internacional. Según las informaciones brindadas por parte del OIJ (2013), esto se debe al hecho de que las organizaciones locales, que comienzan vendiendo drogas en comunidades vulnerables, pueden evolucionar y entrar a formar parte de la cadena de comercio internacional de drogas. Por ejemplo, pueden desenvolver actividades de transporte de drogas dentro del país por parte de grupos de narcotráfico internacional y, sucesivamente, pueden pasar a tener un papel más importante en el trasiego de drogas, sea por vía marítima que terrestre, hacia otros países de la región centroamericana o hacia Estados Unidos y Europa.

Un estudio publicado hace una década por Flores y González Ruiz (2008) nos muestra que, en comparación a los demás países centroamericanos, Costa Rica es el que cuenta con una menor presencia de criminalidad organizada. Sin embargo, la situación está cambiando rápidamente y, desde el 2010, en el país se ha estado verificando un progresivo aumento de la criminalidad organizada Según muestran las estadísticas oficiales, entre las organizaciones delictivas que han sido desarticuladas por parte de las fuerzas de policía nacionales, las internacionales pasaron de ser del 13,3\% del total en el 2010, a ser el 46,6\% en el 2015 y el 35\% en el 2016 (ICD 2016; ICD 2017). Para una mejor comprensión de estos datos, sería importante conocer: las modalidades de clasificación, por parte de las autoridades, de los grupos criminales entre locales e internacionales; cómo y por cuántas personas se conforman los mismos; dónde realizan sus actividades criminales y las diferencias en relación a las formas de violencia que practican.

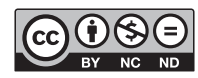


Un primer elemento de distinción entre estos grupos puede ser la nacionalidad de los criminales arrestados, que cuando resultan ser extranjeros, provienen sobre todo de México, Colombia y de países europeos; conforman pequeñas células delictivas y se ocupan de hacer los pedidos de drogas y de supervisar la actividad de desplazamiento de las mismas (OIJ 2013). Esta última actividad suele ser realizada por parte de grupos de apoyo logístico compuestos por sujetos de origen costarricense que se posicionan a un nivel inferior en la cadena de mando, los llamados «transportistas», los cuales tienen la responsabilidad de almacenar la mercancía en el territorio nacional para en un segundo momento, llevarla afuera de las fronteras costarricenses.

En general, la droga que circula en el territorio nacional, tanto para uso interno como para su exportación hacia otros países, es de origen extranjera. La producción nacional se limita al cultivo de marihuana, que por lo general es de muy baja calidad y no está destinada al comercio internacional; mientras que la marihuana de importación para el consumo local proviene principalmente de Colombia y Jamaica (ICD 2013).

En relación al narcotráfico, hay algunos elementos sobre los cuales un gran número de publicaciones concuerdan (Calderón Umaña 2010; 2012; 2013; 2018; Chacón Echeverría y Zúñiga Rodríguez 2015; 2016; ICD 2009; 2010; 2013; 2017; Muñoz Guillén 1999; OIJ 2013; Palma Campos 2011; Pérez Sáinz et al. 2015; Salazar Sánchez y Pérez Sáinz 2015). En primer lugar, el hecho de que Costa Rica en particular y Centroamérica en general, poseen una posición geográfica privilegiada para el transporte internacional de drogas. La cercanía de Costa Rica con los países productores de cocaína del hemisferio sur y la presencia de dos extensas costas (el Mar Caribe y el Océano Pacífico), permitieron que, para las organizaciones del narcotráfico internacional, Costa Rica desempeñe la función de «puente» entre los países de mayor oferta y demanda de esta droga. Además, la posición geográfica de nuestro país y el aumento de los controles ejercidos en las fronteras de los países productores de cocaína a partir de los años 1990 en el marco de la «guerra a las drogas», promovida e impulsada por parte de los Estados Unidos, representó para los narcotraficantes una razón ulterior para substituir las tradicionales rutas aéreas de la cocaína hacia los Estados Unidos y Europa con rutas marítimas y terrestres, aumentando de esta manera la importancia de Costa Rica en el narcotráfico internacional. Villegas Herrera (2014) afirma que esta transformación tuvo un impacto directo en toda la región centroamericana, en la cual se decomisa el 12\% de la cocaína a nivel mundial, con un énfasis mayor en Panamá, Costa Rica y Nicaragua donde se llevan cabo el 90,09\% de estos decomisos. Costa Rica, después de Panamá, es el segundo país de Centroamérica que decomisa más cocaína (OIJ 2013; Villegas Herrera 2014), con una media de 17 toneladas por año entre el 2005 y el 2012 (OIJ 2013). A través del análisis de las publicaciones encontradas, queda claro que Costa Rica ya no desempeña simplemente una función de tránsito de la cocaína y que, progresivamente, se ha vuelto también un país de almacenaje de la misma. El aumento de mercancía que atraviesa el país y el reforzamiento de las relaciones entre los grandes cárteles internacionales y los transportistas locales, permitieron que los primeros comenzaran a pagar a los segundos con droga, ya no únicamente con dinero. Este elemento favoreció el multiplicarse de los micro mercados locales, la competencia y los conflictos entre bandas radicadas en comunidades a lo largo del país 5 .

En relación con la violencia provocada por parte de las organizaciones que se dedican al narcotráfico en Costa Rica, aparecen pocos datos en las publicaciones analizadas. Los escasos elementos que tenemos a disposición (OIJ 2013) nos muestran que, cuanto mayor es el nivel de organización de los grupos que se dedican a este tipo de actividades, menor es el nivel de conflicto que ponen en práctica. Por esta razón, el mayor número de luchas por el control de rutas de comercio suceden entre grupos que transportan marihuana desde Jamaica hacia las costas del caribe costarricense, los cuales son mucho menos organizados que aquellos que se dedican al tráfico de cocaína a través del Océano Pacífico.

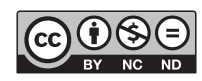

Rev. Reflexiones 98 (2) Julio-Diciembre, 2019: 23-38, E-ISSN: 1659-2859 
A diferencia de las guerras por el control de territorios urbanos vulnerables, que se dan principalmente entre los grupos que se dedican al narcomenudeo, en los países centroamericanos los conflictos entre grupos de narcotraficantes se dan sobre todo en las áreas fronterizas, y Costa Rica no es una excepción (Calderón Umaña 2013).

\section{La organización del narcomenudeo}

A partir de los años noventa, en los barrios marcados por altos niveles de exclusión social de Costa Rica, se verifica un aumento del narcomenudeo y, en consecuencia, también de la violencia a este relacionada (Chacón y Zúñiga 2016). Como se explicó previamente, dicha transformación se debe en parte al aumento de droga que empezó a circular en las comunidades vulnerables a raíz del papel que Costa Rica asumió en el narcotráfico internacional. Aunque no hay manera de verificarlo a través de las publicaciones analizadas, se podría suponer que el aumento del narcomenudeo coincidió con la evolución de pandillas juveniles hacia formas más organizadas de bandas criminales ${ }^{6}$.

Desde esos años, la organización de narcomenudeo ha ido evolucionando y actualmente entre los grupos locales se cuenta también con la presencia de las «narcofamilias»; es decir, «estructuras de corte familiar» $\operatorname{OIJ}(2013,26)$. La mayoría de los grupos criminales que a nivel local se dedican al narcomenudeo, cuentan con una escala jerárquica que se divide de la siguiente manera: en la parte más alta se encuentra la persona «líder» que puede tener contactos directos con proveedores extranjeros o nacionales. Seguidamente está la persona «contadora» quien es una persona de confianza que se encarga de las finanzas. Las personas «supervisoras» se encargan de vigilar la venta de droga. El papel de las personas «operacionales» es intrínsecamente más violento dado que, además de garantizar la seguridad de quien pertenece a sus organizaciones, tienen que reclamar pagos no cumplidos y combatir la competencia en las localidades donde ejercen la venta de drogas. A esta información, se añade la brindada por Calderón Umaña y Salazar Sánchez $(2015,75)$, quienes afirman que, en las comunidades, la venta directa se lleva a cabo «en puntos fijos como casas o centros de venta (búnkeres), puntos semifijos (espacios diversos de la comunidad) o bien, los llamados "puntos móviles" que implican el desplazamiento del vendedor casi a cualquier lugar sugerido por el comprador». Además, estos autores señalan la existencia de proveedores y distribuidores intermedios, los cuales abastecen de droga a las personas narcomenudeantes locales. Quienes proveen se diferencian de quienes distribuyen porque tienen un contacto directo con los cárteles internacionales de la droga. Para complementar el cuadro, Chacón Echeverría y Zúñiga Rodríguez $(2015,298)$ nos ayudan a conocer los sujetos que se posicionan en los niveles inferiores de la cadena de comando del narcomenudeo en las comunidades. Los «robots» son jóvenes de sexo masculino, entre los 12 y 15 años, que se encargan de la venta directa de la droga en las esquinas de las calles. Estos sujetos son descritos por parte de las personas autoras como «criaturas esclavas» que obedecen incondicionalmente a las órdenes de sus superiores a cambio de unos pocos miles de colones al día.

Las informaciones disponibles no permiten entender si la descripción que se acaba de dar de las estructuras de los grupos criminales ha quedado inmutada respecto al momento en el que se realizaron las investigaciones citadas; o si estas reflejan la realidad de todas las comunidades vulnerables en las que se cuenta con la presencia activa de grupos que se dedican al narcomenudeo. De la misma manera, las publicaciones analizadas no brindan suficiente información sobre cuáles son estos grupos, dónde se sitúan y qué diferencias y conflictos hay entre cada uno de estos. El único dato que se encontró al respecto es el hecho de que, en Finca San Juan de Pavas, la banda de «Los Polacos» tiene una contienda por el territorio con la de «Los Diablos» (Chacón Echeverría y Zúñiga Rodríguez 2015).

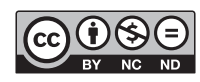

Rev. Reflexiones 98 (2) Julio-Diciembre, 2019: 23-38, E-ISSN: 1659-2859 


\section{Narcomenudeo: control territorial, conflictos armados y represión del Estado}

Los trabajos existentes en el contexto costarricense no consiguen brindar claridad sobre el tema del control territorial ejercido por parte de los grupos criminales que se dedican al narcomenudeo en las áreas urbano-marginalizadas. Esto porque, hasta el momento, en la academia costarricense no ha sido un objeto de análisis per se y, además el fenómeno, como se demuestra a continuación, se encuentra en transformación. En el 2013, el OIJ $(2013,21)$ afirmó que en Costa Rica «los grupos delictivos predadores o vinculados al territorio, claramente presentes en otros países de la región, operan de manera diferente». Y que los grupos criminales costarricenses no «pretenden controlar el territorio, y la mayoría de ellos no está en competición directa» OIJ (2013, 24). Pérez Sáinz (2015) nos recuerda que, en El Salvador los barrios que cuentan con la presencia de las maras, la violencia contra el patrimonio y contra las personas es controlada por parte de estas. En cambio, en los barrios vulnerables de Costa Rica se verifica un mayor nivel de estas formas de violencia, propio por la ausencia de grupos criminales que controlen el territorio y brinden protección a sus residentes. Otra función desarrollada por los grupos criminales, cuando tienen el control de las comunidades, es la mediación de conflictos entre vecinos. Calderón Umaña (2018) afirma que este tipo de actividad no se da en los barrios vulnerables de Costa Rica.

Sin embargo, en el mismo documento del OIJ (2013) citado previamente, se afirma la posibilidad de que dichos grupos evolucionen a formas más organizadas y que, por intereses económicos, lleguen a controlar territorios urbanos e incluso a «convertirse en grupos subestatales presentando formas alternativas de gobernanza» (OIJ 2013, 27). La predicción parece transformarse en realidad, ya que pocos años después Chacón Echeverría y Zúñiga Rodríguez $(2015 ; 2016)$ publicaron dos artículos, en los cuales describen dinámicas generadas por parte de grupos criminales en Pavas y que son propias del control territorial. En estos trabajos se menciona que el monopolio de la violencia, el control de la micro-criminalidad (como por ejemplo los robos y asaltos) y de la violencia doméstica, ahora son parte de las prerrogativas de los grupos criminales, y ya no del Estado. Dichas actividades son desarrolladas por parte de estos grupos principalmente por dos motivos: en primer lugar, para garantizar un nivel suficiente de «paz social», que permita llevar a cabo la venta de narcóticos sin inconvenientes; $y$, en segundo lugar, para proteger los residentes y así aumentar la legitimidad de sus acciones ante estas personas. A modo de ejemplo, una observación de Chacón Echeverría y Zúñiga Rodríguez (2016) sobre Pueblo Nuevo de Pavas nos permite entender que, en relación al último punto, los grupos que se dedican al narcomenudeo controlan los robos y los homicidios para evitar la presencia de policías y la consecuente pérdida de clientes. Esta breve consideración nos sugiere que existe un nivel de control territorial criminal en barrios urbano-marginalizados de Pavas y nos hace pensar que tal control no es total, ya que no consigue mantener alejadas del todo las fuerzas de policía. Sin embargo, dado que las publicaciones citadas mencionan elementos propios de control territorial, pero no lo analizan detalladamente, se necesita de investigaciones específicas relacionadas a este tema que expliquen cuál es la situación actual en las comunidades marginalizadas de nuestro país.

Con base en una investigación desarrollada en Brasil, Colombia y Jamaica, Arias (2017) elabora una división de los grupos que, a nivel de barrio, se dedican al narcomenudeo. En el nivel más bajo de organización, control territorial y conexión con representantes del Estado, se encuentran los grupos que más se involucran en conflictos armados a nivel micro-territorial y que instauraran lo que el autor llama un régimen de «desorden criminal». En el nivel más alto, en cambio, están los que menos hacen uso de la violencia homicida, ya que detienen el control del territorio y mantienen alianzas estables con el Estado. En Costa Rica, donde los homicidios vinculados a la criminalidad organizada pasaron de representar el 2,5\% del total de los homicidios dolosos en el 2010, a un 46,2\% en el 2016 (COMESCO 2017), se puede

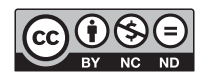


hacer una hipótesis preliminar basada en el modelo de análisis propuesto por Arias (2017). Se puede suponer, por ejemplo, que tal aumento corresponde a la multiplicación de grupos criminales pertenecientes al nivel más bajo de organización, los cuales no consiguen controlar el territorio ni construir alianzas con el mundo de la política institucional y tampoco con las fuerzas de policía. Para entender si el aumento de conflictos armados y homicidios es el producto de la multiplicación de grupos criminales con tales características, es preciso realizar una investigación más amplia, que intente entender también sus causas y consecuencias sociales.

En general, el uso de las armas en el narcomenudeo sirve para proteger la mercancía y el mercado; es decir, para asegurarse el monopolio sobre la venta de drogas en una determinada localidad (Calderón Umaña y Salazar Sánchez 2015). Según el OIJ (2013), los conflictos armados entre las organizaciones del narcomenudeo se dan principalmente por ajustes de cuentas debidos a «tumbes» (robos) de grandes cantidades de droga. Acorde a las estadísticas oficiales, los ajustes de cuentas llegaron a ser 266 en el 2016, mientras en el 2010 fueron 8. Además de los conflictos por ajustes de cuentas, entre los conflictos armados más comunes están los que se producen por «cambios de mando», es decir, cuando una organización criminal es desarticulada por parte de las fuerzas de policía, o uno de sus líderes muere o es arrestado. En esos momentos queda un vacío de poder que suele ser conquistado a través de las armas. El sicariato, es decir, el homicidio «por encargo, a cambio de una compensación económica» (Carrión 2008, 1) es la modalidad más frecuente con la que se asesinan los miembros de bandas rivales del narcomenudeo en Costa Rica. El fenómeno del sicariato en el país es reciente, pues empieza a verificarse de manera sistemática en las estadísticas a inicios de los años 2000 (Mata y Solano Fernández 2006); y conoce un fuerte aumento entre el 2005 y el 2006, cuando se pasa de 6 a 26 casos en un año (ICD 2010). Entre los sicarios, muchos son hombres jóvenes y en algunos casos no llegan a ser mayores de edad, además, no necesariamente pertenecen a las organizaciones del narcomenudeo, ya que pueden ser contratados por estas únicamente para que lleven a cabo homicidios puntuales (Chacón Echeverría y Zúñiga Rodríguez 2015; 2016; ICD 2010; OIJ 2013).

Hasta el momento, la respuesta del Gobierno nacional ante los fenómenos relacionados a la venta de drogas ilícitas ha consistido, sobre todo, en reforzar el aparato represivo contra la micro criminalidad callejera, en particular contra los delitos relacionados al narcomenudeo (Huhn 2012). Según Calderón Umaña $(2010,59)$, esto ha llevado a la implementación de políticas de «mano dura» que no están «orientadas por un principio de justicia y restauración sino por un deseo de venganza». Como consecuencia, en 1994 el Gobierno aumentó la pena máxima de prisión de 25 a 50 años (Calderón Umaña y Gónzalez 2010). Para Solís Moreira (2012), dichas políticas buscan un consenso con la parte de la población que, guiada por alarmes mediáticos, considera como una prioridad el aumento de la represión contra la criminalidad. En particular, en el marco de la «guerra a las drogas», el narcotráfico y narcomenudeo se han vuelto temas centrales para la opinión pública del país, facilitando de esta manera el endurecimiento de las penas para los delitos relacionados a drogas (ICD 2017). Para 2009, con respecto al 2003, las condenas por infracciones a la Ley de Psicotrópicos incrementaron en catorce veces, alcanzando 75.905 casos (PNUD 2010), representando así el 12,8\% del total; llegando a ser la segunda categoría de condenas más elevada después de aquellas relativas a los delitos contra la propiedad (ICD 2016). Como resultado de lo descrito hasta el momento, la población carcelaria tuvo un aumento del $60 \%$ entre el 2005 y el 2015 (Programa Estado de la Nación 2017).

En relación a la acción de las fuerzas de policía para contrarrestar las organizaciones criminales vinculadas al narcotráfico y al narcomenudeo, vale la pena mencionar que, entre el 2010 y el 2016, la Policía de Control de Drogas del Ministerio de Seguridad Pública desarticuló alrededor de 123 de estas organizaciones por año (ICD 2017). Sin embargo, una vez desarticulados, estos grupos pasan a

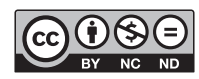

Rev. Reflexiones 98 (2) Julio-Diciembre, 2019: 23-38, E-ISSN: 1659-2859 
ser fácilmente substituidos por otros que ocupan el mismo lugar en la venta o transporte de drogas, fenómeno conocido como «resiliencia delictiva» del narcotráfico y del narcomenudeo (COMESCO 2017; ICD 2016).

\section{Narcomenudeo y violencia: Causas sociales}

Fuera de nuestro país existe un debate vivaz sobre las causas sociales que facilitan la formación de grupos armados que ejercen formas de control territorial en zonas urbano-marginalizadas. Aunque no hay un acuerdo unánime entre las personas investigadoras sobre este punto, la mayoría de los trabajos que se han producido concuerdan sobre el hecho de que el abandono del Estado en estas áreas juega un papel fundamental (Koonings y Kruijt 2004). De hecho, la ausencia de las instituciones gubernamentales «crea un vacío de poder que es aprovechado por otros actores, en este caso las pandillas juveniles, para controlar territorios y monopolizar el uso de la fuerza» (Pérez Sáinz et al. 2016, 10). En muchos casos, el vacío de poder se refleja en la ausencia o ineficacia de las fuerzas policiacas en las zonas urbanas de mayor exclusión social, provocando un sentimiento de inseguridad entre las personas que residen en estas áreas. En muchas ciudades latinoamericanas, los grupos que se dedican al narcomenudeo se han aprovechado de esta situación para ofrecer a los residentes protección contra agresiones externas, así como mecanismos de mediación de conflictos entre vecinos y para dictar las condiciones con las cuales la acción de agencias del Estado y del sector privado suministran diferentes tipos de servicios dentro de las comunidades (Pérez Sáinz 2015).

Es indudable que, en comparación con los países del triángulo norte de América Central, la presencia del Estado en las áreas urbanas vulnerables de Costa Rica ha sido, históricamente, mucho mayor. Por esta razón, junto a Perú, Costa Rica es el único país latinoamericano donde «los barrios populares no se transformaron en enclaves de violencia» (Alba Vega y Kruijt 2017, 494) de manera sistemática. Sin embargo, se ha demostrado que, desde finales de los años 1990, en Costa Rica la mayoría de las personas condenadas por homicidios, es decir el 75,5\%, son residentes de barrios caracterizados por altos niveles de pobreza y exclusión social (Sáenz Rojas 2006). Estadísticas más recientes nos muestran que, si se toma en consideración la generalidad de los delitos, la mayoría de la población penitenciaria de nuestro país se caracteriza por provenir de los estratos socioeconómicos más bajos, por estar desempleada o por desempeñar actividades laborales en el sector informal de los servicios al momento del arresto (Programa Estado de la Nación 2017).

Tomando en consideración la tasa de homicidios del 2007, Huhn (2012) demuestra que la mayor parte de los homicidios se da en San José, la capital y provincia más poblada del país. Sin embargo, el autor hacer notar también que Limón tiene la tasa de homicidios más elevada. Otras publicaciones llegan a los mismos resultados, aunque analizan periodos temporales diferentes, lo que demuestra que existe una constante en la distribución geográfica de los homicidios (Calderón Umaña 2010; COMESCO 2016; 2017; Observatorio de la Violencia 2014b; Sáenz Rojas 2006; Solís Moreira 2017; Vargas Sanabria et al. 2009). Este dato es de particular importancia si se considera que Limón es la provincia que posee los niveles más bajos de desarrollo humano y está entre los más altos niveles de pobreza y desempleo (Sáenz Rojas 2006); y ahí, uno de cada dos homicidios está relacionado a la venta de narcóticos (COMESCO 2017). Huhn (2012) sugiere que, para analizar la violencia letal e intentar individuar sus causas, es necesario observar de manera más detallada las estadísticas y comprender si existe una coincidencia entre las localidades más afectadas por este tipo de crimen y aquellas caracterizadas por tener los índices mayores de exclusión socioeconómica. Para Solís Moreira (2017), la acumulación de desventajas sociales tiene un impacto directo en la distribución geográfica de la criminalidad. De hecho, un estudio

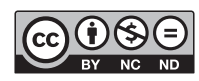

Rev. Reflexiones 98 (2) Julio-Diciembre, 2019: 23-38, E-ISSN: 1659-2859 
reciente (Sánchez Hernández 2018) muestra que los distritos de Costa Rica que presentan mayores tasas de homicidios se encuentran entre los que poseen también los mayores niveles de pobreza y desempleo. Según esta investigación, existe también una relación directa entre los homicidios y la narcoactividad, dato que indica que los distritos en los cuales se llevan a cabo el mayor número de homicidios coinciden con aquellos en los que se decomisan mayores cantidades de droga. Es posible afirmar entonces, que la falta de empleo y las motivaciones de tipo económico facilitan el ingreso de residentes de barrios vulnerables en grupos que se dedican al narcomenudeo (Calderón Umaña 2018; Pérez Sáinz 2015). Para Chacón Echeverría y Zúñiga Rodríguez $(2015 ; 2016)$ el narcomenudeo representa una estrategia de subsistencia e, incluso, de supervivencia para muchos de los jóvenes de estas localidades.

Es importante señalar que el $64 \%$ de las mujeres que ingresan en el sistema penitenciario lo hacen por delitos contra la Ley Sobre Estupefacientes, en particular por venta directa. El hecho que estas mujeres se encuentren en el «último escalón en la pirámide» de la venta de drogas (Hernández Chévez 2013, 76), no sólo comporta que estén en una posición de vulnerabilidad con alto riesgo de ser arrestadas, también refleja su condición socio económica al momento de la detención, cuando «el 37.5\% eran pobres y un $47.5 \%$ se encontraban en extrema pobreza» (Palma Campos ${ }^{7}$ 2011, 254).

La mayor parte de las personas condenadas por delitos relacionados a drogas, son jóvenes entre los 20 y 39 años (ICD 2016); y el número de menores de edad que son privados de su libertad por las mismas razones está en continuo aumento (Rojas et al. 2004; OIJ 2013). La falta de escolaridad entre esta población representa uno de los mayores factores de riesgo en relación al ingreso en el mundo del narcomenudeo y la puesta en práctica de la violencia que lo caracteriza (Observatorio de la Violencia 2010). La relación entre deserción escolar y violencia parece demostrarse si se piensa que los distritos que poseen un mayor nivel de escolaridad tienen también los menores índices de homicidios del país (Sánchez Hernández 2018).

Muchas investigaciones señalan que, a nivel mundial, la desigualdad social tiene un impacto mayor que la pobreza sobre la evolución de la violencia y la criminalidad (Briceño-León 2002; Moser y McIlwaine 2004). Podría entonces tranquilizarnos el hecho de que, como recuerda Calderón Umaña (2018), Costa Rica tiene uno de los índices de desigualdad menores de todo Latinoamérica, llegando incluso a ser el país menos desigual en 1994 (López Sedó 2018). Sin embargo, desde los años 1990, en Costa Rica la desigualdad está en continuo aumento. Además, si se toman en consideración todos los países de América Latina, entre el 2010 y el 2014 la desigualdad aumentó sólo en Costa Rica, Venezuela y en menor medida, en Paraguay (Leitón 2017). Preocupa aún más saber que en el 2016, el país regresó a tener los mismos niveles de desigualdad que tenía en el 1980 (López Sedó 2018), elemento que demuestra un sustancial cambio de marcha en la situación económica del país.

Además de las causas del narcomenudeo y la violencia relacionada, y que se han mencionado hasta ahora, en los textos analizados se hallaron otras más: el consumo de drogas, la circulación de armas de fuego y la masculinidad hegemónica. Obviamente, el primero es una consecuencia de la venta de drogas, pero se vuelve también una causa de la violencia en el momento que los usuarios cometen robos y asaltos para poder pagar sus dosis de consumo personal (Calderón Umaña 2006). Además, se ha verificado una coincidencia entre los cantones del país que tienen los más elevados índices de consumo de drogas, con los que tienen las más altas tasas de homicidios (OIJ 2013). En relación a las armas de fuego, a partir del 2006 y con variaciones leves por cada año, estas son usadas entre el $60 \%$ y $70 \%$ de los homicidios dolosos cometidos en Costa Rica (Observatorio de la Violencia 2011; 2014b; Solís Moreira 2017; Vargas Sanabria et al. 2009). No se encontraron datos relativos a la cantidad de homicidios relacionados a la criminalidad organizada que fueron cometidos con armas de fuego. No obstante, el hecho que estos son llevados a cabo principalmente mediante actos de sicariato, nos hace pensar que

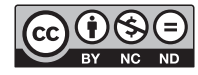

Rev. Reflexiones 98 (2) Julio-Diciembre, 2019: 23-38, E-ISSN: 1659-2859 
son casi la totalidad. El hecho de que, en Costa Rica, la mayoría de los homicidios son llevados a cabo por parte de hombres y tienen como victimarios a otros hombres (Sáenz Rojas 2006; Vargas Sanabria et al. 2009), nos hace pensar que existe una relación entre el crimen violento y la construcción social de la masculinidad hegemónica, la cual exalta la violencia y su uso en la resolución de los conflictos (Bejarano Orozco y Sáenz Rojas 2000). Para Chacón Echeverría y Zúñiga Rodríguez (2016, 72), los conflictos entre bandas y la modalidad con la que se hace un recurso cotidiano a la violencia entre los jóvenes narcomenudeantes, son «performances de masculinidad hegemónica». Si para reafirmar su propia masculinidad, los hombres que se dedican al narcomenudeo deben continuamente poner en acto prácticas violentas, no sorprende que entre el 2015 y el 2016 el 96,8\% de los homicidios dolosos relacionados a la venta de drogas fuera realizado por hombres (COMESCO 2017).

\section{Conclusiones}

Gracias a la recolección y análisis de la bibliografía, fue posible conocer qué se ha escrito en relación a la narcoviolencia en Costa Rica y cuáles son los principales vacíos en la literatura científica sobre este tema ${ }^{8}$. Entre las publicaciones tomadas en consideración, muy pocas tienen como objeto principal de análisis el narcomenudeo y el narcotráfico. La Organización Mundial de la Salud considera que una tasa de homicidios, superior a 10 por cada 100.000 habitantes, corresponde a una situación que es catalogada como epidemia (Observatorio de la Violencia 2014a). En nuestro país se superó este umbral en el 2008 (UN Office on Drugs and Crime's International Homicide Statistics s.f.), y desde ese momento la violencia entre bandas criminales en zonas urbano-marginalizadas ha recibido una mayor atención en el debate público. La escasez de estudios sobre estos temas en Costa Rica podría explicarse entonces a partir de que solo en años recientes se han alcanzado niveles preocupantes de violencia homicida vinculada a la criminalidad organizada.

Las ciencias sociales costarricenses todavía ignoran del todo, o en gran parte, factores que han sido analizados en otros países latinoamericanos como, por ejemplo: el fenómeno de la «narco cultura», es decir, aquella cultura que se crea dentro de los grupos que se dedican al narcotráfico y al narcomenudeo y que permite que en la sociedad se acepte la existencia de estos grupos. Otros temas han sido poco estudiados en Costa Rica. Por ejemplo, no se ha realizado ninguna investigación ad hoc que busque comprender cuál es la relación entre la narcoviolencia y el creciente nivel de desigualdad. Además, se encontró que hacen falta investigaciones y publicaciones académicas que expliquen de manera sistemática y exhaustiva cuál es el papel de las fuerzas de policía en la prevención y represión de la violencia relacionada a la venta de drogas ilícitas.

Una investigación ulterior podría ayudarnos también a comprender mayormente cuál es el nivel de control territorial ejercido por grupos que se dedican al narcomenudeo, y cuál es la relación entre los miembros de las comunidades y los de dichos grupos. En particular, trabajos futuros podrían ponerse los siguientes objetivos: a) identificar cuáles son los factores que provocan los conflictos entre estos grupos o que permiten que los mismos sean evitados; b) verificar si los grupos criminales regulan la vida cotidiana y asociativa de las comunidades e identificar qué acciones ponen en práctica para alcanzar dicho resultado; c) verificar si hay grupos criminales que consiguen mantener el monopolio de la violencia y de los mercados ilícitos, e identificar qué acciones ponen en práctica para alcanzar dicho resultado; d) describir las formas de violencia derivadas de los conflictos relacionados al narcomenudeo y el impacto que estas tienen en la vida de los habitantes de la comunidad y e) reconstruir la historia del control territorial entre bandas criminales que se dedican al narcomenudeo y de otras formas de conflicto y de violencia a este relacionados.

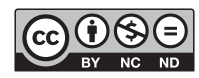

Rev. Reflexiones 98 (2) Julio-Diciembre, 2019: 23-38, E-ISSN: 1659-2859 
Para poder comprender las dinámicas y particularidades locales de la narcoviolencia en Costa Rica, es necesario producir datos primarios que nos permitan analizar el fenómeno y, si es posible, proponer soluciones específicas a los problemas locales. En particular, se señala la necesidad de investigaciones de tipo cualitativo, posiblemente de etnografías, ya que la mayoría de las menciones que se hacen a los temas analizados, se basan en datos secundarios, en particular en las estadísticas criminales. Se subraya la importancia de producir datos georreferenciados y más segregados, respecto a los que actualmente se disponen desde las publicaciones analizadas sobre los homicidios y, en particular, sobre aquellos relacionados a las actividades de narcotráfico y narcomenudeo, en las cuales se describe la distribución de los mismos por provincias, cantones, y distritos. No obstante, es necesario poder ubicar la localización de los homicidios también por barrios. En efecto, en un mismo distrito se pueden encontrar barrios con condiciones socioeconómicas y tasa de homicidios muy diferentes entre sí, y al mismo tiempo, conocer la localidad exacta de los homicidios y otras formas de violencia nos podría permitir comprender mejor dónde se ubican los focos de los conflictos entre las bandas criminales, así como sus causas y consecuencias.

\section{Notas}

1. El número de homicidios en Costa Rica está en continuo aumento. Sin embargo, es mucho menor que el de otros países de la región centroamericana. Es suficiente saber que, en el 2015 en El Salvador, la tasa de homicidios era de 109 cada 100.000 habitantes (World Bank s.f.).

2. A través del programa de análisis de datos cualitativos NVivo 11, el material se dividió en 147 códigos, cada uno de los cuales corresponde a uno de los elementos que conforman el conocimiento existente sobre el fenómeno analizado.

3. La mayoría de los documentos, 34 de 55, aparecen en revistas y libros publicados en Costa Rica. Siete se publicaron en Chile (5 son capítulos que pertenecen al mismo libro), 3 en México, 2 en España, 2 en libros publicados en los Estados Unidos e Inglaterra y una publicación por cada uno de los siguientes países: Alemania, Canadá, Colombia, Cuba, Ecuador, Holanda e Inglaterra.

4. De estas 88 publicaciones, 34 fueron descartadas porque no presentan datos importantes para el presente estudio. La mayor parte de estas, por ejemplo, son boletines estadísticos del Instituto Costarricense sobre Drogas, lo cuales se limitan a describir la fluctuación de las estadísticas criminales por trimestre.

5. El cambio en la paga de dinero a droga y el efecto que tuvo en las comunidades vulnerables se menciona en varias publicaciones (Chacón y Zúñiga 2015; ICD 2010; 2013; 2017; OIJ 2013; Palma Campos 2011; Pérez Sáinz 2015), sin embargo, aunque no hay motivos para dudar de su veracidad, en ninguna de estas se cita la fuente originaria o se explica qué elementos se tienen para demostrar que así fue.

6. En los textos tomados en consideración -así como sucede con el narcomenudeo y el narcotráficolos conceptos de pandilla, banda y organización criminal muchas veces suelen ser usados como sinónimos, dificultando ulteriormente el análisis del fenómeno de la narcoviolencia. Algunas publicaciones intentan desambiguar estos conceptos e introducen una distinción ulterior en la cual, además de las pandillas - o cuadrillas para Rodríguez Aguilar (2017) -, se incluyen las «barras», es decir grupos que cometen delitos menores y se sitúan en una posición intermedia entre las

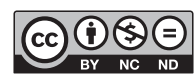

Rev. Reflexiones 98 (2) Julio-Diciembre, 2019: 23-38, E-ISSN: 1659-2859 
pandillas y las bandas (Cruz y Carranza 2006 cit. en Rodríguez Aguilar 2017; Rodríguez Aguilar y Solano Acuña 2011; Solís 2008).

7. El estudio de Palma Campos $(2011,257)$ nos permite comprender que la mujer que participa activamente en el narcomenudeo dice hacerlo principalmente por motivos relacionados a su maternidad (la mayoría son jefas de hogares), reafirmando de esta manera una «subjetividad de género construida en función de los «otros» y no de sí misma».

8. Además de los temas tratados en este artículo, se analizaron también las respuestas institucionales al narcotráfico, al narcomenudeo y en general, al crimen organizado. En particular, se encontró que hacen falta investigaciones y publicaciones académicas que expliquen, de manera sistemática, cuál es el papel de las fuerzas de policía en la prevención y represión de tales fenómenos; y no se halló producción académica o institucional sobre la influencia que, convenciones, leyes y políticas internacionales de control de drogas, tienen en el contexto costarricense.

\section{Referencias}

Alba Vega, Carlos, y Dirk Kruijt. 2017. «Viejos y nuevos actores violentos en América Latina: temas y problemas». Foro Internacional 47 (julio-setiembre): 485-516. http://www.jstor.org/ stable/27738841

Arias, Enrique Desmond. 2017. Criminal Enterprises and Governance in Latin America and the Caribbean. Virginia, Estados Unidos: Cambridge University Press. https://doi.org/10.1017/9781316650073

Asamblea Legislativa de la República de Costa Rica. 1998. Ley sobre estupefacientes, sustancias psicotrópicas, drogas de uso no autorizado, actividades conexas, legitimación de capitales y financiamiento al terrorismo. Costa Rica: Sistema Costarricense de Información Judicial. http://www. cicad.oas.org/fortalecimiento_institucional/legislations/PDF/CR/ley_8204.pdf

Bejarano Orozco, Julio, y Mario Alberto Sáenz Rojas. 2000. «Consumo de drogas y muerte violenta en Costa Rica». Adicciones 12: 435-441. http://www.adicciones.es/index.php/adicciones/article/ viewFile/654/643

Briceño-León, Roberto. 2002. «La nueva violencia urbana en América Latina». Sociologías 4(juliodiciembre): $34-51$.

Calderón Umaña, Rodolfo. 2006. «El delito en Costa Rica: una propuesta analítica». Revista Centroamericana de Ciencias Sociales 3 (julio): 83-121.

- 2010. «Violencia social en Centroamérica: Desafíos y perspectivas para la gobernabilidad y la convivencia democrática». Revista Centroamericana de Ciencias Sociales 7: 53-72. https://ucr. academia.edu/RodolfoCalderonUmana

- 2012. Delito y cambio social en Costa Rica (Primera). San José: FLACSO. http://www.flacso.or.cr/ images/flippingbook/pdfs/libros/delito_cambio_social.pdf

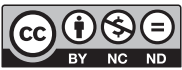

Rev. Reflexiones 98 (2) Julio-Diciembre, 2019: 23-38, E-ISSN: 1659-2859 
- 2013. «Proposiciones analíticas para el estudio de la violencia en Centroamérica: una mirada desde la exclusión social». Revista Digital de La Maestría En Ciencias Penales 0: 199-226. http://revistas. ucr.ac.cr/index.php/RDMCP/article/view/12443

- 2018. «Violence and social exclusion in urban contexts in Central America». En Social theories of urban violence in the Global South, editado por Jennifer Erin Salahub, Markus Gottsbacher y John de Boer, 99-120. Londres y New York: Routledge Studies in Cities and Development.

Calderón Umaña, Rodolfo. y Eduardo González. 2010. «Costa Rica». En Crime and punishment around the world, editado por Janet P. Stamatel y Hung-En Sung, 109-117. California, Estados Unidos: ABC-CLIO.

Calderón Umaña, Rodolfo, y Karla Salazar Sánchez. 2015. «Dinámicas de violencia en las comunidades costarricenses». En Exclusión social y violencias en territorios centroamericanos (Primera), editado por Juan Pablo Pérez Sáinz, 61-98. San José, Costa Rica: FLACSO. http://209.177.156.169/ libreria_cm/archivos/pdf_1274.pdf

Carrión, Fernando. 2008. «Sicariato». Boletín Ciudad Segura 24: 4-9.

Chacón Echeverría, Laura, y Jimmy Zúñiga Rodríguez. 2015. «¿Será vida ver la muerte tan de cerca? Conflictos de drogas y lucha espacial en Pavas, Costa Rica». Anuario de Estudios Centroamericanos, 41: 279-302. https://doi.org/10.15517/aeca.v41i1.21849

- 2016. «Prácticas espaciales y tráfico de drogas en Pueblo Nuevo de Pavas: reterritorializar La Línea». Revista de Ciencias Sociales, 2: 69-88. https://doi.org/10.15517/rcs.v2i152.27352

COMESCO (Comisión Técnica Interinstitucional sobre Estadísticas de Convivencia y Seguridad Ciudadana). 2016. Compendio de investigaciones relacionadas con la convivencia y seguridad ciudadana en Costa Rica. San José: COMESCO. http://sisvi.mj.go.cr/meshcms/themes/sisvi/Doc/ Compendio_Comesco.pdf

- 2017. Análisis de los homicidios dolosos vinculados a la delincuencia organizada durante el período 2010-2016. San José: COMESCO. http://www.cr.undp.org/content/dam/costa_rica/docs/undp_cr_ analisis homicidios 2010-2016 FINAL.pdf

Cruz, José Miguel, y Marlon Carranza. 2006. «Pandillas y políticas públicas: el caso de El Salvador». En Juventudes, violencia y exclusión. Desafíos para las políticas públicas, editado por Javier Moro, 133-171. Guatemala: INDES.

Flores, Carlos, y Samuel González Ruiz. 2008. «Democracia y crimen organizado». En organizado en América Latina y el Caribe, editado por Luis Guillermo Solís y Francisco Rojas Aravena, 51-94. Santiago, Chile: FLACSO.

Hernández Chévez, Lang Ying. 2013. «Rutas críticas hacia la participación en el narcotráfico de mujeres privadas de libertad en Liberia, Guanacaste». InterSedes, 14: 70-89. http://www.intersedes.ucr. ac.cr/ojs/index.php/intersedes/article/view/368

Huhn, Sebastian. 2012. Criminalidad y discurso en Costa Rica (Primera). Traducido por Anne Stahn. San José: FLACSO. http://www.democraciaycooperacion.net/IMG/pdf/CriminalidadCR.pdf

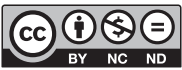

Rev. Reflexiones 98 (2) Julio-Diciembre, 2019: 23-38, E-ISSN: 1659-2859 
ICD (Instituto Costarricense sobre Drogas). 2009. Mujeres infractoras a la ley de psicotrópicos recluidas en el Centro de Atención Institucional el Buen Pastor. San José: ICD. http://www.icd.go.cr/ portalicd/images/docs/uid/investigaciones/Investigacion Buen Pastor.pdf

- 2010. Situación nacional sobre drogas y actividades conexas 2010. San José: ICD. http://www.icd. go.cr/portalicd/images/docs/uid/informes/Situacion_Nacional_Drogas2010.pdf

- 2012. Incidencia de los homicidios dolosos atribuibles a la delincuencia organizada en la tasa de homicidios por cada 100000 habitantes. Años 2000 al 2010. San José: ICD. http://www.icd.go.cr/ portalicd/images/docs/uid/investigaciones/Tasa_homicidios_dolosos_2012.pdf

- 2013. Plan Nacional sobre drogas, Legitimación de Capitales y Financiamiento al Terrorismo 2013-2017. San José: Gobierno de Costa Rica. http://www.icd.go.cr/portalicd/images/docs/icd/ PND_2013_2017_mar_2015.pdf

- 2016. Informe de situación nacional sobre drogas y actividades conexas. Costa Rica 2015. San José: Gobierno de Costa Rica. http://www.icd.go.cr/portalicd/images/docs/uid/informes/SituacionNac/ CR_SitNacional_DrogasActivCon_2016.pdf

- 2017. Informe de situación nacional sobre drogas y actividades conexas. Costa Rica 2016. San José: Gobierno de Costa Rica. http://www.icd.go.cr/portalicd/images/docs/uid/informes/SituacionNac/ CR_Situacion_Nacional_Drogas_ActivConexas_2017.pdf

Koonings, Kees y Dirk Kruijt. 2004. Armed actors. Organized violence and state failure in Latin America. Londres, Inglaterra: Z. Books.

Leitón, Patricia. 2017. «Desigualdad baja en América Latina pero sube en Costa Rica». La Nación, 31 de julio. https://www.nacion.com/economia/politica-economica/desigualdadbaja-en-america-latina-pero-sube-en-costa-rica/B2IFQJRWA5F3XNTXE4L6B2CFNE/ story/

López, Leonardo Raffo y Diego Gómez Calderón. 2017. «Redes criminales y corrupción en la era del microtráfico y el narcomenudeo». Revista de Economía Institucional, 19: 227-261.

López Sedó, Valeria. 2018. «La odiosa desigualdad nos atrasó cuarenta años». Semanario Universidad, 13-19 de junio. https://semanariouniversidad.com/pais/costa-o-rica-la-odiosa-desigualdad -que-nos-devolvio-a-1980/

Mata, Leonardo y Mario Solano Fernández. 2006. «Homicidio doloso en Costa Rica, 1993-2005: magnitud, tipología y tasas por país de origen del imputado». Población y Salud En Mesoamérica, 4 (julio-diciembre): 1-17. https://revistas.ucr.ac.cr/index.php/psm/article/view/4560

Moser, Caroline y Cathy Mcllwaine. 2004. Encounters with violence in Latin America. Urban poor perceptions from Colombia and Guatemala. London: Psychology Press.

Muñoz Guillén, Mercedes. 1999. «Narcotráfico, democracia y soberanía nacional en Costa Rica». Anuario de Estudios Centroamericanos 25: 33-47. https://revistas.ucr.ac.cr/index.php/anuario/ article/view/1939/1903

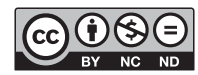


Observatorio de la violencia. 2010. Informe 5. Proyectos Sociales y Comunitarios Desarrollados desde el Observatorio Nacional de la Violencia. Costa Rica: Observatorio de la Violencia.

- 2011. Informe estadístico 6. Armas de fuego y violencia en Costa Rica. San José: Observatorio de la Violencia.

- 2014a. Informe 12. Tendencias en el comportamiento de los homicidios en Costa Rica. Período 2000-2013. San José: Observatorio de la Violencia. http://www.mjp.go.cr/Documento/ DescargaDIR/1045

- 2014b. Muertes violentas en Costa Rica: homicidio, femicidio, suicidio, muertes por accidentes de tránsito. Período 2008-2013. San José: Observatorio de la Violencia.

OIJ (Organismo de Investigación Judicial). 2013. Tráfico de drogas y amenazas del crimen organizado en Costa Rica. San José: OIJ. http://www.icd.go.cr/portalicd/images/docs/uid/informes/UID_ Anuario2012_Control_Oferta.pdf

Palma Campos, Claudia. 2011. «Delito y sobrevivencia: las mujeres que ingresan a la cárcel El Buen Pastor en Costa Rica por tráfico de drogas». Anuario de Estudios Centroamericanos, 37: $245-270$.

Parakilas, Jacob. 2012. «The Complexities of Narcoviolencia: Understanding the Mexican Drug Conflict as a Market of Violence». Interdisciplinary Political Studies, 2 (marzo): 36-47.

Pérez Sáinz, Juan Pablo. 2015. «Reflexiones sobre territorios marginales urbanos en Centroamérica». En Exclusión social y violencias en territorios centroamericanos, editado por Juan Pablo Pérez Sáinz, 19-60. San José, Costa Rica: FLACSO.

Pérez Sáinz, Juan Pablo, Rodolfo Calderón Umaña y Gilbert Brenes Camacho. 2016. «Exclusión social, violencia y ámbito doméstico. Evidencia y reflexiones desde Centroamérica». Papeles de Población 87: 9-41.

Pérez-Sáinz, Juan Pablo, Larissa Brioso, Rodolfo Calderón Umaña, Margarita Montoya, Karla Salazar, Mario Zetino, Eric Hershberg y Daniel Esser. 2015. Violence and Community Capabilities: Insights for Building Safe and Inclusive Cities in Central America. Costa Rica y El Salvador: Center for Latin American \& Latino Studies.

PNUD (Programa de las Naciones Unidas para el Desarrollo). 2010. Política integral y sostenible de seguridad ciudadana y promoción de la paz social. San José: PNUD. https://www.oas.org/dsp/ documentos/politicas_publicas/polsepaz_costa_rica.pdf

Programa Estado de la Nación. 2017. Causas de la privación de libertad y perfil de la población penitenciaria. Costa Rica: Segundo Informe Estado de La Justicia. http://www.estadonacion.or.cr/ justicia/assets/estado-de-la-justicia-1-baja.pdf

Rodríguez Aguilar, Onésimo. 2017. Aquí está todo: ratas, evolución y honor. Cuadrillas juveniles y barrio en Costa Rica. San José, Costa Rica: Arlekín.

Rodríguez Aguilar, Onésimo y Ana Sofía Solano Acuña. 2011. «Pandillas, violencia y dinámicas socioculturales en la Costa Rica urbana». Intersticios Sociales, 1: 1-42.

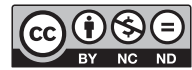

Rev. Reflexiones 98 (2) Julio-Diciembre, 2019: 23-38, E-ISSN: 1659-2859 
Rojas, Marielos, Sandra Jiménez, y Miryan Cruz. 2004. La violencia social en Costa Rica. San José, Costa Rica: Organización Panamericana de la Salud.

Sáenz Rojas, Mario Alberto. 2006. «Los homicidios dolosos en Costa Rica: caracterización sociodemográfica». Revista de Ciencias Sociales, 1-2: 177-188.

Salazar Sánchez, Karla y Juan Pablo Pérez Sáinz. 2015. «Estrategias de confrontación ante algunas formas de violencia. La experiencia de dos territorios costarricenses». En Exclusión social y violencias en territorios centroamericanos, editado por Juan Pablo Pérez Sáinz, 145-190. San José, Costa Rica: FLACSO. http://209.177.156.169/libreria_cm/archivos/pdf_1274.pdf

Sánchez Hernández, Leonardo. 2018. Patrones territoriales y factores sociodemográficos asociados a los homicidios y el narcotráfico en Costa Rica. San José. https://www.icd.go.cr/portalicd/images/ docs/uid/investigaciones/Patrones_territoriales_homicidios_narcotrficoCR18.pdf

Solís Moreira, Julio. 2012. «Políticas de la representación, etiquetamiento criminal y responsabilización por la seguridad ciudadana en editoriales del Diario Extra (2008-2010)». Rupturas, 2: 36-57.

Solís Moreira, Julio. 2017. «Reflexiones sociológicas acerca de la violencia delictiva y su medición en Costa Rica». Revista Digital de La Maestría En Ciencias Penales, 9: 1-31. https://revistas.ucr. ac.cr/index.php/RDMCP/article/view/29674

Solís, Luis. Guillermo. 2008. «Pandillas juveniles y gobernabilidad democrática en América Latina y el Caribe». En Crimen organizado en América Latina y el Caribe, editado por Luis Guillermo Solís y Francisco Rojas, 139-174. Santiago, Chile: FLACSO.

UN Office on Drugs and Crime's International Homicide Statistics. (s.f.). «Intentional homicides (per 100,000 people». https://data.worldbank.org/indicator/VC.IHR.PSRC.P5?locations=CR

Vargas Sanabria, Maikel, Leslie Solano Calderón y Raúl Bonilla Montero. 2009. «Análisis médico legal de los homicidios en Costa Rica en el 2008». Medicina Legal de Costa Rica 26 (setiembre): 7-27. http://www.scielo.sa.cr/scielo.php?script=sci_arttext\&pid=S1409-00152009000200002

Villegas Herrera, César. 2014. «La economía ilícita del narcotráfico en Centroamérica. Análisis del mercado de la cocaína y las respuestas políticas de los Estados». Anuario de Estudios Centroamericanos 40: 245-262. http://revistas.ucr.ac.cr/index.php/anuario/article/download/16641/16146

The World Bank. (s.f.). «Intentional homicides (per 100,000 people)», acceso el 10 de agosto del 2018, https://data.worldbank.org/indicator/VC.IHR.PSRC.P5

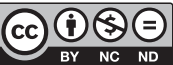

